\title{
Anomalous Spectral Shift of Near- and Far-Field Plasmonic Resonances in Nanogaps
}

\author{
Anna Lombardi, ${ }^{\dagger}$ Angela Demetriadou, ${ }^{\ddagger}, \S$ Lee Weller, ${ }^{\dagger}$ Patrick Andrae, ${ }^{\dagger, \perp}$ Felix Benz, ${ }^{\dagger}$ \\ Rohit Chikkaraddy, ${ }^{\dagger}$ Javier Aizpurua, ${ }^{\ddagger}$ and Jeremy J. Baumberg, ${ }^{* \dagger}$ \\ ${ }^{\dagger}$ NanoPhotonics Centre, Cavendish Laboratory, University of Cambridge, Cambridge, CB3 0HE, U.K. \\ ${ }^{\ddagger}$ Centro de Física de Materiales, Centro Mixto CSIC-UPV/EHU, and Donostia International Physics Center (DIPC), Paseo Manuel \\ Lardizabal 4, 20018 Donostia-San Sebastián, Spain \\ ${ }^{\S}$ Blackett Laboratory, Department of Physics, Imperial College London, London SW7 2AZ, United Kingdom
}

\section{Supporting Information}

ABSTRACT: The near-field and far-field spectral response of plasmonic systems are often assumed to be identical, due to the lack of methods that can directly compare and correlate both responses under similar environmental conditions. We develop a widely tunable optical technique to probe the nearfield resonances within individual plasmonic nanostructures that can be directly compared to the corresponding far-field response. In tightly coupled nanoparticle-on-mirror constructs
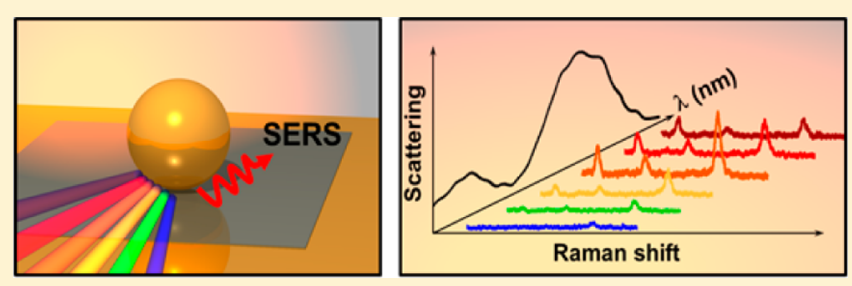
with nanometer-sized gaps we find $>40 \mathrm{meV}$ blue-shifts of the near-field compared to the dark-field scattering peak, which agrees with full electromagnetic simulations. Using a transformation optics approach, we show such shifts arise from the different spectral interference between different gap modes in the near- and far-field. The control and tuning of near-field and far-field responses demonstrated here is of paramount importance in the design of optical nanostructures for field-enhanced spectroscopy, as well as to control near-field activity monitored through the far-field of nano-optical devices.

KEYWORDS: SERS, ultrafast tunable lasers, plasmonics, nanoparticle on mirror, sensing

$\mathrm{T}$ he interaction of light with noble metal nanostructures excites collective electron oscillations in the form of localized plasmonic resonances. As a result, such plasmonic nanostructures are able to confine light within extremely small volumes, millions of times smaller than a wavelength-sized box. Squeezing light into such small regions creates near-thousandfold field enhancements, which are ideal for intense surfaceenhanced Raman scattering (SERS), thus allowing only a few atoms, molecules, or nano-objects to be directly tracked. ${ }^{1}$ So far, researchers have typically assumed that both the localized near-field and radiated far-field support their resonant behavior (i.e., strongest field enhancements) at similar spectral wavelengths. As a result, optimization of SERS has depended on measurements of the far-field scattering spectrum.

Here we show that when the optical field is tightly confined by nanoscale gaps, the resulting multiple order plasmon resonances supported at different wavelengths interfere with each other differently to build up the signal from the near- and far-fields. As a result, significant spectral shifts are observed. We experimentally demonstrate this using a spectral-scanning technique that simultaneously records dark-field scattering spectra and tunable-pump SERS measurements on each nanostructure individually. We utilize plasmonic constructs for this that provide extremely robust nanoscale gaps, ${ }^{2}$ using $\mathrm{Au}$ nanoparticles separated from a bulk $\mathrm{Au}$ film by an ultrathin molecular spacer, known as the nanoparticle-on-mirror
(NPoM) geometry. ${ }^{3-5}$ In contrast to the red-shifts always found in isolated nanoparticles, ${ }^{6}$ the near-field NPoM resonance from SERS is found to be always blue-shifted from the scattering peak. We explain this through a transformation optics model that allows the decomposition of the total signal into individual modes that show different radiative properties. In particular the $n=1$ and 2 modes interfere constructively in the far-field, but destructively in the near-field. From this understanding, our experiments also allow us to show that the SERS background arises from a completely different process from the SERS vibrational signal, as it follows instead the farfield spectral enhancement. Our insights provide a solid intuition to predict how near-fields behave within a wide variety of plasmonic nanoconstructs.

Direct measurements of the near-field plasmonic enhancement spectra either are probe-based techniques or must exploit nonlinear processes, since only then do the evanescent fields contribute most strongly. Probe-based techniques are not suitable for single NPoM measurements, and second-harmonic generation is not very reliable for this task, as it possesses both bulk and surface contributions and is thus very sensitive to many additional aspects of nanoscale geometry. Thirdharmonic generation techniques ${ }^{7}$ are also possible but so far

Received: December 10, 2015

Published: February 8, 2016 
are primarily single-wavelength studies. ${ }^{8-12}$ The other favorable process for this task is SERS, but this has also been challenging because of the requirement for wide tuning of the Raman pump laser, while ensuring high-contrast tunable filtering of the scattered light from the background Rayleigh pump scatter. As a result, most experiments work with arrays of nanoparticles ${ }^{13}$ or use a limited number of excitation wavelengths on individual nanostructures. ${ }^{14-18}$ Alternative approaches with fixed excitation wavelength that attempt to tune the plasmon resonance suffer uncontrolled changes in confinement and enhancement. ${ }^{8-12}$ Recent experiments ${ }^{15}$ have managed to deliver wavelength-scanned Raman and dark-field measurements on lithographically defined plasmonic dimers in order to ascertain how quantum tunneling affects the SERS amplitude. Lithography however generates considerable uncertainty in the gap sizes and morphologies. Compared to such nanoparticle dimers, the NPoM geometry guarantees much better control of gap size between gold film and nanoparticle, higher reproducibility, and a much simpler and robust nanoassembly procedure and has thus been recently utilized in many experimental studies. ${ }^{3-5}$ The well-defined architecture also precisely defines the orientation of the optical fields and of the molecules that are currently studied in SERS, and thus allows precise comparison of the near- and far-field response.

Our experimental setup is optimized to realize both dark-field microscopy and broadband-tunable SERS measurements on the same single nanoparticle at the same time (Figure 1a). For darkfield scattering spectroscopy white light is focused on the sample through a high numerical aperture $(\mathrm{NA}=0.8) 100 \times$ objective, and a cooled spectrometer detects the scattering of single nanoparticles, which are kept well spatially separated (coverage $<1 \mu \mathrm{m}^{-2}$ ).

To realize broadband-tunable SERS a sub-nanometer linewidth-tunable laser source is required. To create this, a $200 \mathrm{fs}$ Ti:sapphire oscillator pumps a femtosecond optical parametric oscillator to give tunable output over visible and near-infrared wavelengths from 500 to $1040 \mathrm{~nm}$ (see Methods). The output is spectrally narrowed below $1 \mathrm{~nm}$ using an acousto-optic programmable dispersive broadband filter (AOPDF), ${ }^{19}$ yielding fully automated tuning with multi-milliwatt output powers. This Gaussian beam is focused in an inverted microscope to a diffraction-limited spot using the same $100 \times$ objective. For each excitation wavelength, Rayleigh-scattered light is filtered out using a computer-controlled translatable custom-built array of multiple linear variable long wave pass (LP) filters with an overall optical density (OD) of 11, maximum transmission of $80 \%$, and cutoff spectral width of $10 \mathrm{~nm}$. Stokes Raman signals are recorded with a spectrometer and cooled EMCCD camera. Calibration on bulk solids confirms this system is capable of Raman measurements across the entire visible and infrared.

For the near-field measurements here, a $p$-terphenylthiol (TPT) self-assembled molecular monolayer (SAM) is used as a nanoscale spacer between the flat gold surface and $60 \mathrm{~nm}$ gold nanoparticles placed on top. The gap thickness, which depends on the orientation angle of the molecules on the gold surface, is found to be $d=1.4 \pm 0.1 \mathrm{~nm}$ through phase-modulated ellipsometry measurements, in good agreement with previous work. ${ }^{1}$ Individual nanoparticles are first optically characterized by dark-field spectroscopy (Figure $1 \mathrm{~b}$ ), which shows for all spectra a strong coupled plasmon mode resonance in the nearinfrared around $730 \pm 20 \mathrm{~nm}$. The $<20 \mathrm{~nm}$ (fwhm) variation in spectral peak arises from the $10 \%$ distribution of $\mathrm{Au}$ nanoparticle diameters. The tightly confined hot spot created (a)

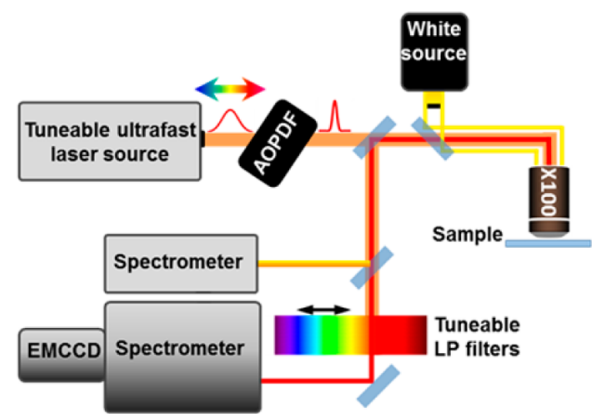

(b)
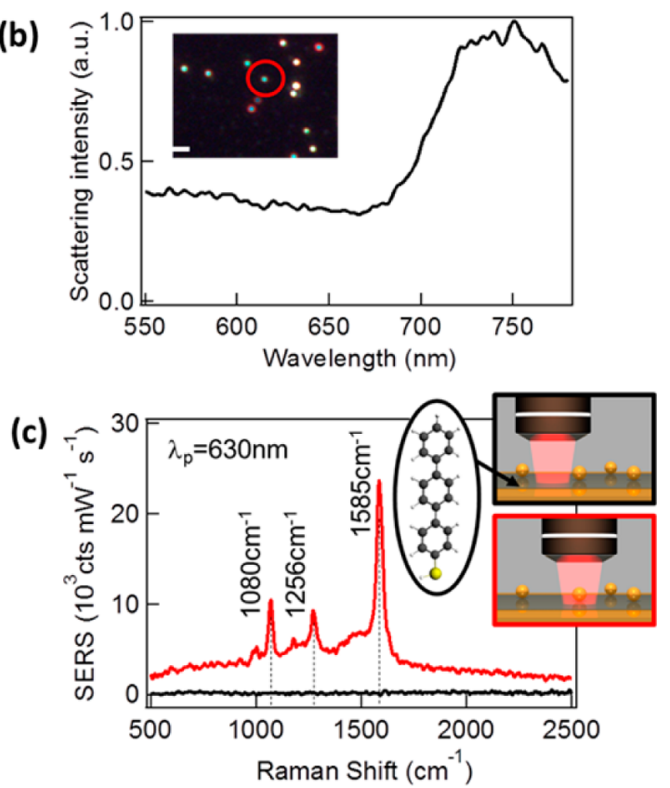

Figure 1. (a) Broadband-tunable SERS spectroscopy coupled with dark-field microscopy for single-nanoparticle studies, based on an AOPDF spectral filter. (b) Scattering spectra from a single $60 \mathrm{~nm}$ gold nanoparticle on a gold mirror. A TPT self-assembled molecular monolayer is used as a spacer. Inset: Dark-field image of a sample highlighting a nanoparticle under study (red circle); scale bar is $1 \mu \mathrm{m}$. (c) Surface-enhanced Raman spectra measured on (red) and off (black) a single nanoparticle. Laser excitation wavelength is $630 \mathrm{~nm}$. Inset: Gold nanoparticles on a gold substrate, separated by a $1.4 \mathrm{~nm}$ TPT molecular spacer.

within the gap (lateral size $(R d)^{1 / 2} \approx 6.5 \mathrm{~nm}$ ) is then an ideal situation to compare near- and far-field spectra.

When the laser (at pump wavelength $\lambda_{\mathrm{p}}=630 \mathrm{~nm}$ in Figure 1 ) is focused away from any Au nanoparticle, the Raman scattering from the molecular SAM is below our detection noise level (Figure 1c, black). However, focusing on an NPoM elicits hundreds of times stronger optical fields, greatly enhancing the SERS signal of TPT molecules confined within the plasmonic nanogap (Figure 1c, red). Three dominant vibrational lines are seen corresponding to a $\mathrm{C}-\mathrm{H}$ rocking mode $\left(1080 \mathrm{~cm}^{-1}\right)$ and to in-plane stretching of the benzene rings (1256 and 1585 $\mathrm{cm}^{-1}$ ). The average laser power at the sample is kept below 1 $\mu \mathrm{W}$, which is needed to avoid any shifts in the NPoM dark field spectra or any changes in SERS over time (Supporting Information). Similar SERS signals are obtained from each NPoM.

By scanning the laser wavelength, we measure the plasmonicinduced SERS enhancement from TPT to access the near-field spectrum of the NPoM (Figure 2a). The vibrational peaks do not shift with pump $\lambda_{\mathrm{p}}$, but their amplitudes show strong 


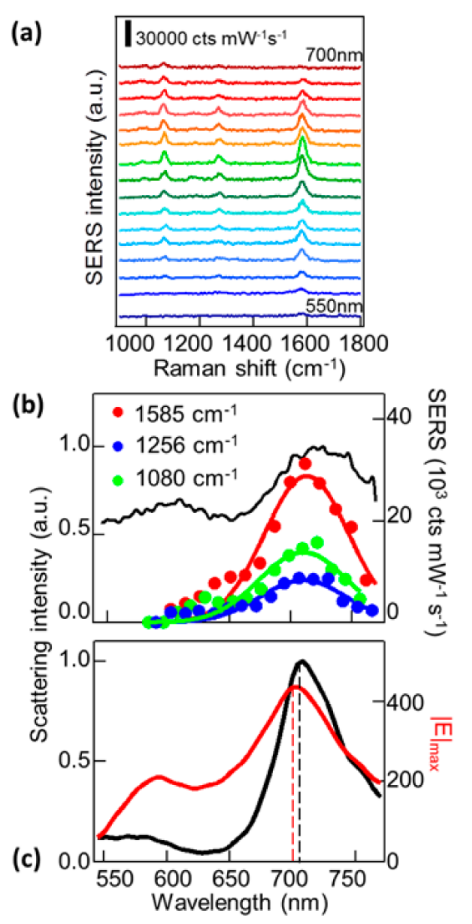

Figure 2. (a) SERS spectra from a single $60 \mathrm{~nm}$ nanoparticle tuning the excitation laser from $\lambda_{\mathrm{p}}=550$ to $700 \mathrm{~nm}$ in $10 \mathrm{~nm}$ steps. (b) Extracted SERS intensity of three TPT Raman peaks vs SERS peak (outgoing) wavelength, compared to the normalized scattering spectrum (black) of the same single gold NPoM (lines show Gaussian fits). (c) FDTD simulations of scattering (black) and maximum nearfield (red) for a $60 \mathrm{~nm}$ gold NPoM placed on a gold mirror.

enhancements at $\lambda_{\mathrm{p}}=640 \mathrm{~nm}\left(1585 \mathrm{~cm}^{-1}\right.$ mode $), \lambda_{\mathrm{p}}=650 \mathrm{~nm}$ $\left(1256 \mathrm{~cm}^{-1}\right.$ mode $)$, and $\lambda_{\mathrm{p}}=670 \mathrm{~nm}\left(1080 \mathrm{~cm}^{-1}\right.$ mode $)$ (Figure 2a). Control measurements show the plasmonic origin of these resonances and imply SERS enhancement factors of $\sim 10^{8}$ with $N_{\text {SERS }}=200$ molecules confined within each hot spot (see Methods).
Plotting the extracted experimental SERS enhancements $I_{\text {SERS }}\left(\nu, \lambda_{\mathrm{p}}\right)$ of each of the three main TPT peaks against the outgoing wavelength (Figure $2 \mathrm{~b}$ ) shows they reach their maxima close to the coupled mode observed in far-field scattering, but are blue-shifted by $\sim 22 \mathrm{meV}$. This is contrary to the behavior for isolated plasmonic nanoparticles, in which case the nearfield resonance is found to be red-shifted compared to the scattering. ${ }^{6,20-22}$ This red-shift is associated with the damping of a plasmonic resonance. ${ }^{6,22}$ A localized surface plasmon can be interpreted in terms of a driven damped oscillator. When damping is present, the maximum oscillation amplitude occurs at a lower energy than the natural frequency of the oscillator, while maximum dissipation occurs at the natural frequency, giving a spectral red-shift. A more complete description of the oscillator model describing plasmonic resonances was discussed by Kats et al. ${ }^{23}$ Blue-shifted near-field spectra were previously reported $^{24}$ for large nanoparticle arrays. Using single nanostructures here confirms this behavior does not originate from any sample inhomogeneity or periodic effect. Finite difference time domain (FDTD) simulations of the maximum near-field and the scattering far-field optical response of a single NPoM confirm this behavior (Figure 2c) and show it is a general property of all closely coupled plasmonic resonators such as dimers (Supporting Information). We note that the overall spectral shifts between simulation and experiment here probably arise from faceting of the nanoparticle, which increases the coupling and red-shifts the coupled plasmon resonance. ${ }^{25,26}$

Results on a range of NPoMs show that the near-field resonance is always blue-shifted from the far-field resonance by 4 to $55 \mathrm{meV}$ depending on the nanoparticle (Figure 3a). Different sized nanoparticles tune the coupled mode, but in all cases $I_{\text {SERS }}$ follows a Gaussian spectral profile with a $\sim 2$-fold reduction in line width compared to the corresponding resonant plasmonic mode in scattering. This is also unexpected since both dark-field and Raman scattering require each photon to couple in and to couple back out, both resonantly enhanced by the plasmonic antenna.
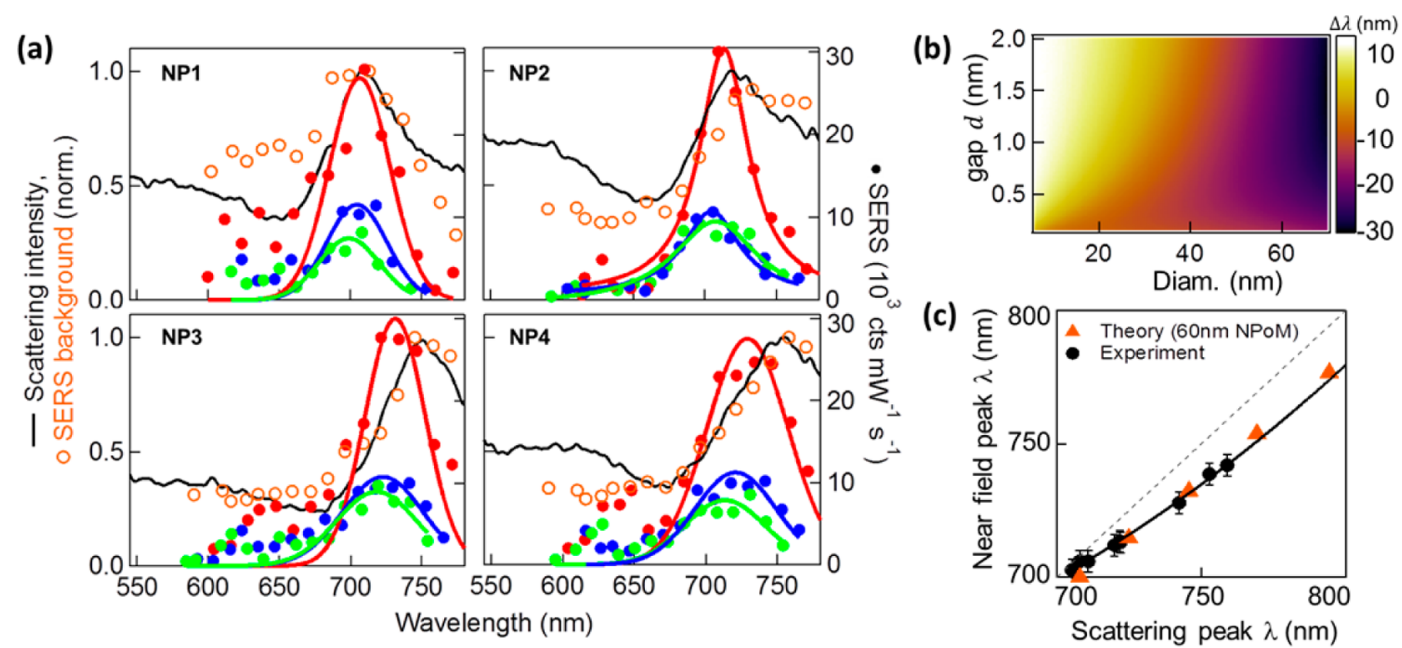

Figure 3. (a) Evolution of SERS intensity of TPT vibrational modes at $1585 \mathrm{~cm}^{-1}$ (red), $1256 \mathrm{~cm}^{-1}$ (green), and $1080 \mathrm{~cm}^{-1}$ (blue) vs emitted Raman wavelength, compared to scattering spectra (black) for four NPoMs (lines show Gaussian fits). Also shown is SERS background around 1585 $\mathrm{cm}^{-1}$ (orange). (b) Calculated resonance shift $\Delta \lambda=\lambda_{\text {near-field }}-\lambda_{\text {scat }}$ for different size nanoparticles in NPoM and different gap sizes. (c) Comparison of wavelengths of peak SERS emission and the dark-field plasmon resonance, for experiments (black) and simulations (orange). Polynomial fit of experimental points is shown as a plain black line. 

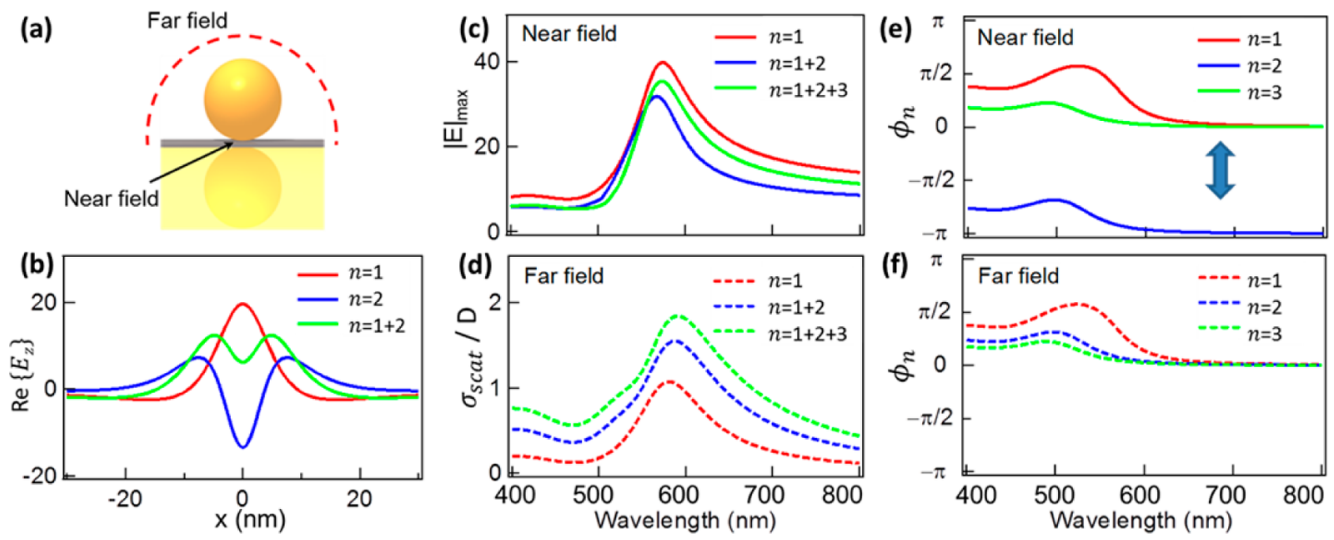

Figure 4. (a) Simulation geometry, giving (b) distribution of fields perpendicular to NP surface for dipolar and quadrupolar modes labeled as $n=1$, 2. (c, d) Spectral dependence of maximum field in the gap (near-field) and scattering (far-field) for first few $n$. $D$ is the diameter of the nanoparticle. (e, f) Phase of $n=1,2,3$ in the far-field (f) and in the center of the gap (near-field, e).

To understand the origin of this effect and the spectral narrowing of the near-field resonance, it is useful to separate out the contributions from each plasmonic resonance supported in the gap, which thus requires further theoretical insights.

Contrary to an isolated nanoparticle, when two plasmonic resonators (here a nanoparticle and mirror) are coupled, the near-field enhancement is heavily dependent on the physical geometry of the nanogap and on the excited modes within it. By implementing a transformation optics technique ${ }^{27}$ (Supporting Information), we model the optical response of the gap modes, both in the near-field and in their radiative emission. The transformation optics technique is developed here in a two-dimensional system to provide the key intuition to understand the nature and composition of the fundamental modes. We find that the dipole-localized surface plasmon polariton resonance $(n=1)$ strongly interferes with the quadrupole mode $(n=2)$ (corresponding field distributions in Figure S6 of the Supporting Information). This leads to similar field patterns around most of the nanoparticle, but pronounced differences inside the gap (Figure $4 a-d$ ). Critically, they have opposite phase, $\phi_{\mathrm{n}}$, in the near-field (i.e., destructive superposition, Figure $4 \mathrm{e}$ arrow), but they radiate coherently to the far-field (i.e., constructive interference, Figure 4f). Hence, the second gap mode shifts the far-field $\left(\sigma_{\text {scat }}\right)$ resonance to longer wavelengths (Figure $4 \mathrm{~d}$ ) and the near-field (SERS) to shorter wavelengths (Figure 4c).

Even higher-order modes become more significant for extremely small separations $(<0.3 \mathrm{~nm}$ for the NPoM discussed here), where the nanoparticle couples even stronger with the mirror. Their phase shifts alternate with even/odd $n$ in the near-field, but all modes add coherently in the far-field, shifting $\sigma_{\text {scat }}$ to even longer wavelengths. Both experiment and simulations confirm this, showing an increased blue-shift for coupled modes that are located further in the infrared. As the nanoparticle moves away from the mirror, the blue-shift observed in the coupled regime decreases until we reach the decoupled regime (isolated nanoparticle) and a red-shift is observed instead (Figure 3b), in agreement with previous results. On the other hand, it has been reported ${ }^{28,29}$ that for geometrically more complex structures (such as trimers), the field enhancement in the near-field shows a maximum "in regions where there is no hint of a resonance in the absorption/ extinction". 29 Such structures support multiple modes at nearby frequencies, which commonly result in complex spectral shifts in the far-field response. Our NPoM system provides a unique opportunity to isolate a precise modal structure and perform a well-defined modal analysis on a robust spectral composition, which is not typical in other SERS systems. It should be noted that all theoretical calculations are performed purely classically, ignoring nonlocality and electron spill-out from the plasmonic metals; the basic concepts here will be little altered by quantum effects for this range of particle-surface separation distances.

Further insights can be extracted from the dependence of the SERS background on $\lambda_{\mathrm{p}}$. This background contribution has been highly debated in the literature, ${ }^{30,31}$ with competing explanations of plasmon luminescence, image molecules, and inelastic electron scattering and from contamination, however, clarifying data are still lacking. For each NPoM we extract the background in the vicinity of the $1585 \mathrm{~cm}^{-1}$ peak and plot it as a function of the emitted wavelength (Figure 3a open circles). This clearly shows the SERS background does not match the spectral shape of the near-field enhancement in the gap but closely follows the far-field optical scattering response of the plasmonic NPoM, in both spectral position and line width. Recent work $^{30,31}$ shows that much of the SERS background must come from optical penetration inside the metal, where it can induce inelastic scattering of the electrons. In the experiments here, molecules are placed only in the gap (hence they probe only the near-fields), while the $n=1,2$ modes localize the light around the entire nanoparticle surface. Hence, our spectral-tuned measurements thus prove that the SERS peaks and background must arise from different sources, as also suggested by super-resolution imaging studies. ${ }^{32}$ As a result, we prove that the SERS background observed here has a component that tracks the far-field enhancement, as well as an equally intense spectrally constant component arising from the surrounding planar substrate.

We have thus shown that the clear identification and spectral separation of the near- and far-field resonances can be achieved using precision spectrally tuned SERS measurements on single nanoparticles. Both experiment and theory agree in the resonance shifts and spectral widths, which are found to be controlled by the coherent superposition between different plasmon gap modes. In the spherical nanoparticle-on-mirror geometry the dominant modes are the $n=1$ and 2, which have opposite phase in the near-field but the same phase in the farfield, resulting in a blue-shift of the SERS peak compared to the 
dark-field scattering and a 2-fold smaller resonance line width. This intuitive understanding of how the resonance positions are determined is generally applicable to coupled plasmonic systems. It also shows that the ever-present SERS background does not come from the same spatial locations as the near-fieldcontrolled SERS peaks.

\section{METHODS}

Sample Preparation. Gold substrates are prepared by evaporating $100 \mathrm{~nm}$ gold (Kurt J. Lesker Company, PVD 200) on a silicon (100) wafer (Si-Mat, Germany) with a rate of $1 \AA$ / s. To obtain atomically smooth films, a standard template stripping method is used: silicon substrates are glued onto the freshly evaporated gold using an epoxy glue (EpoTek 377), ${ }^{33}$ and the resulting gold/epoxy/silicon sandwich is peeled off the silicon wafer.

Self-assembled monolayers of $1,1^{\prime}, 4^{\prime}, 1^{\prime \prime}$-terphenyl-4-thiol (Sigma-Aldrich, 97\%) are formed by submerging the freshly template-stripped substrates into a $1 \mathrm{mM}$ solution in water-free ethanol (Sigma-Aldrich, reagent grade, anhydrous) for $24 \mathrm{~h}$. The samples are subsequently thoroughly rinsed with ethanol and blown dry. Gold nanoparticles (BBI Solutions, UK) are deposited by drop casting from the as-received solution. The deposition time is adjusted in order to obtain the desired nanoparticle coverage. The samples are rinsed with Milli-Q water in order to remove any salt residues.

Ellipsometry. The thickness of the self-assembled monolayers is measured using both ellipsometry (Jobin-Yvon UVISEL spectroscopic ellipsometer) and normalizing plasmon resonance spectroscopy. ${ }^{34}$ For the ellipsometry measurements an angle of incidence of $70^{\circ}$ is used. The data are modeled and fitted using a three-layer model. A thickness of $1.5 \mathrm{~nm}$ is determined with a refractive index of $n=1.45$.

Dark-Field Spectroscopy. Optical dark-field images are recorded on a custom Olympus GX51 inverted microscope. Samples are illuminated with a focused white light source (halogen lamp). The scattered light is collected through a $100 \times$ dark-field objective (LMPLFLN-BD, NA = 0.8) and analyzed with a fiber-coupled (50 $\mu \mathrm{m}$ optical fiber) Ocean Optics QE65000 cooled spectrometer. We use a standard diffuser as a reference to normalize white light scattering. For each sample, we record optical spectra from 20 randomly selected isolated nanoparticles.

Tunable SERS. An ultrafast laser system based on a $200 \mathrm{fs}$ Ti:sapphire oscillator (Spectra Physics MaiTai delivering $200 \mathrm{fs}$ pulses, fwhm $10 \mathrm{~nm}$, at $80 \mathrm{MHz}$ repetition rate) pumps a femtosecond optical parametric oscillator (Spectra Physics Inspire). This light source provides a tunable output over a wide range of visible and near-infrared wavelengths from 500 to $1040 \mathrm{~nm}$. The monochromaticity of the output beam is reduced below $1 \mathrm{~nm}$ spectral bandwidth using an acousto-optic programmable dispersive broadband filter (AOPDF, Dazzler, Fastlite). Relying on interactions between a polychromatic acoustic wave and a polychromatic optical wave in the bulk of a birefringent crystal, it is fully automated across a wide wavelength range (500-900 nm), yielding average output powers of several milliwatts.

SERS experiments are performed on the same modified Olympus GX51 inverted microscope used for dark-field spectroscopy. A monochromatic wavelength-tunable laser beam is focused on the sample using a $100 \times$ objective (NA $=0.8$ ). Raman scattering is collected through the center of the objective and analyzed with a Shamrock SR-303i fully automated spectrometer coupled with an EMCCD camera water cooled to $-85{ }^{\circ} \mathrm{C}$. For the current experiments we use a $6001 / \mathrm{mm} 650 \mathrm{~nm}$ blazed grating. Rayleigh scattering is filtered out with a set of three long pass linear variable filters (DELTA); this filtering system allows the detection of a minimum Raman shift of about $400 \mathrm{~cm}^{-1}$ over the studied spectral range. The system is calibrated using a silicon substrate as a reference. Spectral acquisitions are taken using an integration time of $10 \mathrm{~s}$ and an average laser power on the sample below $1 \mu \mathrm{W}$.

The enhancement factor per molecule (EF) is calculated for each nanoparticle by integrating the Raman peak areas and taking the ratio between SERS at $1585 \mathrm{~cm}^{-1}\left(I_{\text {SERS }}\right)$ and the corresponding unenhanced signal from the bulk powder $\left(I_{\mathrm{R}}\right)$ :

$$
\mathrm{EF}=\frac{I_{\mathrm{SERS}} / N_{\text {SERS }}}{I_{\mathrm{R}} / N_{\mathrm{R}}}
$$

where $N_{\text {SERS }}$ and $N_{R}$ are the estimated number of molecules contributing to SERS and Raman signals, respectively (Supporting Information). From a spot size of $0.4 \mu \mathrm{m}$ and assuming that $N_{\text {SERS }}=200$ molecules are confined in each hot spot, we estimate the measured EF to be $\sim 10^{8}$ for this excitation wavelength. We compare this to predictions from numerical simulations of this geometry, which suggest $\mathrm{EF}=$ $\left|E_{\mathrm{p}}\right|^{2}\left|E_{\text {SERS }}\right|^{2}=10^{6}-10^{7}$, where $\left|E_{\mathrm{p}}\right|$ is the field amplitude enhancement at the incident laser wavelength, while $\left|E_{\text {SERS }}\right|$ is the field amplitude enhancement at the outgoing wavelength (Stokes emission). By fitting Lorentzian lines to each vibrational peak and subtracting the SERS backgrounds, the spectral evolution $I_{\text {SERS }}\left(\nu, \lambda_{\mathrm{p}}\right)$ of the three main TPT peaks is extracted. Normalizing these to the incident laser power (we separately confirm all signals are linear in pump power), these are plotted as a function of the excitation wavelength and directly compared to the dark-field spectrum of the same nanoparticle (Figure $2 \mathrm{~b}$ ).

FDTD Simulation. The electromagnetic response of the nanoparticle on mirror geometry has been simulated by threedimensional FDTD calculations using Lumerical FDTD Solutions v8.9. The structure has been modeled as a gold sphere of $60 \mathrm{~nm}$ diameter on top of a $200 \mathrm{~nm}$ thick gold layer, with a $1 \mathrm{~nm}$ thick dielectric sheet in between. For the gold, we referred to the dielectric constants reported in Johnson and Christy. ${ }^{35}$ The gold nanoparticle was illuminated with $\mathrm{p}$ polarized plane waves from an angle of incidence of $\theta_{i}=55^{\circ}$. The scattered light was then collected within a cone of halfangle $\theta_{c}=53^{\circ}$, based on the numerical aperture of the objective.

\section{ASSOCIATED CONTENT}

\section{S Supporting Information}

The Supporting Information is available free of charge on the ACS Publications website at DOI: 10.1021/acsphotonics.5b00707.

Laser power effects on the optical response of single nanoparticles, terphenylthiol powder absorption measurements, biphenyl-4-thiol-tunable SERS measurements on different size nanoparticles, FDTD simulations for a dimer geometry, transformation optics technique, scanning electron microscopy correlation (PDF)

\section{AUTHOR INFORMATION}

\section{Corresponding Author}

*E-mail: jjb12@cam.ac.uk. 


\section{Present Address}

${ }^{\perp}$ Nanooptical Concepts for PV, Helmholtz-Zentrum Berlin, Hahn-Meitner-Platz 1, 14109 Berlin, Germany.

Notes

The authors declare no competing financial interest.

\section{ACKNOWLEDGMENTS}

We acknowledge financial support from EPSRC grants EP/ G060649/1, EP/L027151/1, EP/G037221/1, EPSRC NanoDTC, and ERC grant LINASS 320503. J.A. acknowledges support from project FIS2013-41184-P from Spanish MINECO and project NANOGUNE'14 from the Department of Industry of the Basque Country. F.B. acknowledges support from the Winton Programme for the Physics of Sustainability. R.C. acknowledges financial support from St. John's College, Cambridge, for a Dr. Manmohan Singh Scholarship. P.A. acknowledges funding from the Helmholtz Association for the Young Investigator group VH-NG-928 within the Initiative and Networking Fund. We thank Laurynas Pukenas and Steve Evans (University of Leeds, UK) for support with the ellipsometry measurements.

\section{REFERENCES}

(1) Kneipp, K.; Wang, Y.; Kneipp, H.; Perelman, L. T.; Itzkan, I.; Dasari, R. R.; Feld, M. S. Single Molecule Detection Using SurfaceEnhanced Raman Scattering (SERS). Phys. Rev. Lett. 1997, 78, 16671670.

(2) Benz, F.; Tserkezis, C.; Herrmann, L. O.; De Nijs, B.; Sanders, A.; Sigle, D. O.; Pukenas, L.; Evans, S. D.; Aizpurua, J.; Baumberg, J. J. Nanooptics of Molecular-Shunted Plasmonic Nanojunction. Nano Lett. 2015, 15, 669-674.

(3) Mubeen, S.; Zhang, S.; Kim, N.; Lee, S.; Kramer, S.; Xu, H.; Moskovits, M. Plasmonic Properties of Gold Nanoparticles Separated from a Gold Mirror by an Ultrathin Oxide. Nano Lett. 2012, 12, 20882094.

(4) Daniels, J. K.; Chumanov, G. Nanoparticle-Mirror Sandwich Substrates for Surface-Enhanced Raman Scattering. J. Phys. Chem. B 2005, 109, 17936-17942.

(5) Mertens, J.; Eiden, A. L.; Sigle, D. O.; Huang, F.; Lombardo, A.; Sun, Z.; Sundaram, R. S.; Colli, A.; Tserkezis, C.; Aizpurua, J.; Milana, S.; Ferrari, A. C.; Baumberg, J. J. Controlling Subnanometer Gaps in Plasmonic Dimers Using Graphene. Nano Lett. 2013, 13, 5033-5038.

(6) Zuloaga, J.; Nordlander, P. On the Energy Shift between NearField and Far-Field Peak Intensities in Localized Plasmon Systems. Nano Lett. 2011, 11, 1280-1283.

(7) Hajisalem, G.; Nezami, M. S.; Gordon, R. Probing the Quantum Tunneling Limit of Plasmonic Enhancement by Third Harmonic Generation. Nano Lett. 2014, 14, 6651-6654.

(8) Driskell, J. D.; Larrick, C. G.; Trunell, C. Effect of Hydration on Plasmonic Coupling of Bioconjugated Gold Nanoparticles Immobilized on a Gold Film Probed by Surface-Enhanced Raman Spectroscopy. Langmuir 2014, 30, 6309-6313.

(9) Liusman, C.; Li, H.; Lu, G.; Wu, J.; Boey, F.; Li, S.; Zhang, H. Surface-Enhanced Raman Scattering of Ag-Au Nanodisk Heterodimers. J. Phys. Chem. C 2012, 116, 10390-10395.

(10) Kang, M.; Kim, J.-J.; Oh, Y.-J. A Deformable Nanoplasmonic Membrane Reveals Universal Correlations Between Plasmon Resonance and Surface Enhanced Raman Scattering. Adv. Mater. 2014, 26, $4510-4514$.

(11) Blaber, M. G.; Schatz, G. C. Extending SERS into the Infrared with Gold Nanosphere Dimers. Chem. Commun. 2011, 47, 37693771.

(12) Lamberti, A.; Virga, A.; Angelini, A.; Ricci, A.; Descrovi, E.; Cocuzza, M.; Giorgis, F. Metal-elastomer Nanostructures for Tunable SERS and Easy Microfluidic Integration. RSC Adv. 2015, 5, 44044410.
(13) McFarland, A.; Young, M. A.; Dieringer, J. A.; Van Duyne, R. P. Wavelength-Scanned Surface-Enhanced Raman Excitation Spectroscopy. J. Phys. Chem. B 2005, 109, 11279-11285.

(14) Li, L.; Hutter, T.; Steiner, U.; Mahajan, S. Single Molecule SERS and Detection of Biomolecules with a Single Gold Nanoparticle on a Mirror Junction. Analyst 2013, 138, 4574-4578.

(15) Zhu, W.; Crozier, K. B. Quantum Mechanical Limit to Plasmonic Enhancement as Observed by Surface-Enhanced Raman Scattering. Nat. Commun. 2014, 5, 5228.

(16) Ye, J.; Hutchinson, J. A.; Uji-i, H.; Hofkens, J.; Lagae, L.; Maes, G.; Borghs, G.; Van Dorpe, P. Excitation Wavelength Dependent Surface Enhanced Raman Scattering of 4-aminothiophenol on Gold Nanorings. Nanoscale 2012, 4, 1606-1611.

(17) Wang, P.; Zhang, D.; Zhang, L.; Fang, Y. The SERS Study of Graphene Deposited by Gold Nanoparticles with 785nm Excitation. Chem. Phys. Lett. 2013, 556, 146-150.

(18) Wei, A.; Kim, B.; Sadtler, B.; Tripp, S. L. Tunable SurfaceEnhanced Raman Scattering from Large Gold Nanoparticle Arrays. ChemPhysChem 2001, 12, 743-745.

(19) Verluise, F.; Laude, V.; Cheng, Z.; Spielmann, C.; Tournois, P. Amplitude and Phase Control of Ultrashort Pulses by Use of an Acousto-Optic Programmable Dispersive Filter: Pulse Compression and Shaping. Opt. Lett. 2000, 25, 575-577.

(20) Chen, J.; Albella, P.; Pirzadeh, Z.; Alonso-Gonzalez, P.; Huth, F.; Bonetti, S.; Bonanni, V.; Akerman, J.; Nogues, J.; Vavassori, P. Plasmonic Nickel Nanoantennas. Small 2011, 7, 2341-2347.

(21) Alonso-Gonzalez, P.; Albella, P.; Neubrech, F.; Huck, C.; Chen, J.; Golmar, F.; Casanova, F.; Hueso, L. E.; Pucci, A.; Aizpurua, J. Experimental Verification of the Spectral Shift Between Near- and FarField Peak Intensities of Plasmonic Infrared Nanoantennas. Phys. Rev. Lett. 2013, 110, 203902.

(22) Moreno, F.; Albella, P.; Nieto-Vesperinas, M. Analysis of the Spectral Behavior of Localized Plasmon Resonances in the Near- and Far-Field Regimes. Langmuir 2013, 29, 6715-6721.

(23) Kats, M. A.; Yu, N.; Genevet, P.; Gaburro, Z.; Capasso, F. Effect of radiation damping on the spectral response of plasmonic components. Opt. Express 2011, 19, 21748.

(24) Dieringer, J. A.; McFarland, A.; Shah, N.; Stuart, D.; Whitney, A. Surface Enhanced Raman Spectroscopy: New Materials, Concepts, Characterization Tools, and Applications. Faraday Discuss. 2006, 132, 9-26.

(25) Sigle, D. O.; Mertens, J.; Herrmann, L. O.; Bowman, R. W.; Ithurria, S.; Dubertret, B.; Shi, Y.; Ying Yang, H.; Tserkezis, C.; Aizpurua, J.; Baumberg, J. J. Monitoring Morphological Changes in 2D Monolayer Semiconductors Using Atom-Thick Plasmonic Nanocavities. ACS Nano 2015, 9, 825-830.

(26) Tserkezis, C.; Esteban, R.; Sigle, D. O.; Mertens, J.; Herrmann, L. O.; Baumberg, J. J.; Aizpurua, J. Hybridization of Plasmonic Antenna and Cavity Modes: Extreme Optics of Nanoparticle-OnMirror Nanogaps. Phys. Rev. A: At., Mol., Opt. Phys. 2015, 92, 053811.

(27) Aubry, A.; Lei, D. Y.; Maier, S. A.; Pendry, J. B. Plasmonic Hybridization between Nanowires and a Metallic Surface: a Transformation Optics Approach. ACS Nano 2011, 5, 3293-3308.

(28) Wustholz, K. L.; Herny, A.-I.; McMahon, J. M.; Freeman, R. G.; Valley, N.; Piotti, M. E.; Natan, M. J.; Schatz, G. C.; Van Duyne, R. P. Structure-Activity Relationships in Gold Nanoparticle Dimers and Trimers for Surface-Enhanced Raman Spectroscopy. J. Am. Chem. Soc. 2010, 132, 10903-10910.

(29) Le Ru, E. C.; Galloway, C.; Etchegoin, P. G. On the connection between optical absorption/extinction and SERS enhancements. Phys. Chem. Chem. Phys. 2006, 8, 3083-3087.

(30) Barnett, S. M.; Harris, N.; Baumberg, J. J. Molecules in the Mirror: how SERS Background Arise From the Quantum Method of Images. Phys. Chem. Chem. Phys. 2014, 16, 6544-6549.

(31) Hugall, J. T.; Baumberg, J. J. Demonstrating Photoluminescence from $\mathrm{Au}$ is Electronic Inelastic Light Scattering of a Plasmonic Metal: The Origin of SERS Background. Nano Lett. 2015, 15, 2600-2604.

(32) Willets, K. A.; Stranahan, S. M.; Weber, M. L. Shedding Light on Surface-Enhanced Raman Scattering Hot Spots through Single- 
Molecule Super-Resolution Imaging. J. Phys. Chem. Lett. 2012, 3, $1286-1294$.

(33) Hegner, M.; Wagner, P.; Semenza, G. Ultrafast Atomically Flat Template-Stripped Au Surfaces for Scanning Probe Microscopy. Surf. Sci. 1993, 291, 39-46.

(34) De Nijs, B.; Bowman, R. W.; Herrmann, L. O.; Benz, F.; Barrow, S. J.; Mertens, J.; Sigle, D. O.; Chikkaraddy, R.; Eiden, A.; Ferrari, A.; Scherman, O. A.; Baumberg, J. J. Unfolding the Contents of sub-nm Plasmonic Gaps using Normalising Plasmon Resonance Spectroscopy. Faraday Discuss. 2015, 178, 185-193.

(35) Johnson, P. B.; Christy, R. W. Optical Constants of the Noble Metals. Phys. Rev. B: Solid State 1972, 6, 4370-4379. 